Bilirubin

\section{Bilirubin, a curse and a boon}

\section{J D Ostrow, C Tiribelli}

\section{Unconjugated bilirubin is a curse at high concentrations, producing apoptosis and cell death, but a boon at more physiological levels, protecting cells against oxidant damage}

B oth hydrophobic bile salts $^{12}$ and unconjugated bilirubin (UCB) ${ }^{3}$ induce apoptosis in cultured cells at moderately elevated concentrations and cell necrosis at higher concentrations. Retention of bile salts in cholestasis is believed to cause secondary damage to hepatocytes, ${ }^{4}$ and retention of UCB in severe neonatal jaundice is known to cause bilirubin encephalopathy. ${ }^{5}$ For both agents, the cytotoxicity results from damage to mitochondrial membranes, with collapse of the transmembrane potential and generation of a mitochondrial membrane permeability transition, ${ }^{12} 6$ and ursodeoxycholic acid or its amidates can prevent apoptosis by inhibiting this process. ${ }^{127}$ It is therefore surprising that, as reported by Granato et al in this issue of Gut, ${ }^{8}$ UCB, at concentrations far above those known to be cytotoxic to neurones and astrocytes, $^{3}$ is protective against apoptosis induced in cultured rat hepatocytes by the hydrophobic bile salt glycochenodeoxycholate (GCDC) [see page 1774]. This finding raises interesting questions concerning whether UCB is a curse or a boon, and why various organs and tissues have such different susceptibilities to toxicity from these substances.

\section{EXPERIMENTAL CONSIDERATIONS}

Like many previous in vitro studies of the cytotoxicity of $\mathrm{UCB}^{9}$ and bile salts, ${ }^{10}$ the studies of Granato and colleagues ${ }^{8}$ were performed at concentrations of the unbound fraction far above those that are clinically relevant. Although the incubation media contained $18 \mu \mathrm{M}$ bovine serum albumin, concentrations of UCB and GCDC greatly exceeded the high affinity binding capacity of $1 \mathrm{~mol} / \mathrm{mol}$ albumin. The final unbound concentrations of UCB $\left(\mathrm{B}_{\mathrm{f}}\right)$ were also far above aqueous saturation, ${ }^{11}$ so the pigment must have been heavily self aggregated. ${ }^{12}{ }^{13}$ Unbound concentrations of GCDC were also well above those seen in plasma in cholestasis, questioning the relevance of the results to clinical conditions. ${ }^{10}$ Furthermore, although UCB was recrystallised, polar derivatives had not been extracted with alkali, ${ }^{14}$ and surface active impurities, which may have contributed to the toxicity, were not removed from GCDC by available methods. ${ }^{15} 16$

Bilirubins were once regarded as toxic wastes, of importance only when excessively high levels of UCB led to the development of encephalopathy. ${ }^{5}$ It is now appreciated that bilirubins and biliverdin are extremely potent antioxidants, ${ }^{17}{ }^{18}$ and that these antioxidant properties may be cytoprotective, ${ }^{19} 20$ even at physiological concentrations. A new finding of the Granato paper $^{8}$ was that the synthetic ditaurate conjugate of bilirubin, a satisfactory surrogate for the natural glucuronosyl conjugates, was nearly as potent as UCB in protecting hepatocytes against toxicity from GCDC.

The antioxidant effects of bilirubins and biliverdin are mediated largely by sacrificial oxidation of these pigments, ${ }^{21}$ consuming reactive oxygen species that could damage membrane lipids. However, UCB also regenerates other natural antioxidants, ${ }^{18}$ and traps a variety of reactive oxygen species. ${ }^{22}$ Binding to plasma albumin, ${ }^{22}$ and possibly to cytosolic proteins, ${ }^{23}$ enhances the antioxidant potency of UCB. Thus both bound and unbound UCB and conjugated bilirubins in plasma and the cytosol are available to protect cell membranes from lipid peroxidation. By contrast, it is only "free" UCB $\left(B_{f}\right)$, the $<0.1 \%$ of total plasma UCB that is not protein bound, ${ }^{24}{ }^{25}$ that can enter cells by passive or facilitated diffusion ${ }^{26}$ that determines its toxic effects intracellularly. ${ }^{24}$

A recent meta-analysis of in vitro studies conducted at relevant $B_{\mathrm{f}}$ levels revealed that cultured neurones and astrocytes suffer both plasma membrane and mitochondrial damage at $B_{f}$ levels slightly to modestly above the $70 \mathrm{nM}$ aqueous solubility limit of UCB; ${ }^{3}$ this suggests that the inimical species might be soluble oligomers and/or small colloidal aggregates of UCB. By contrast, exposure to $\mathrm{UCB}$ at $\mathrm{B}_{\mathrm{f}}$ levels below

\section{IS BILIRUBIN A CURSE OR A BOON?}

$70 \mathrm{nM}$ protects cultured neurones and their mitochondria against oxidative stress induced by hydrogen peroxide; this protection is diminished at higher $B_{f}$ values where the toxic effects of UCB became dominant. ${ }^{20}$

Low levels of endogenous UCB, generated intracellularly by microsomal haeme oxygenases, likewise protect against oxidative damage to cells. ${ }^{19}$ 27-30 This raises a seeming paradox-how can such low (nanomolar) concentrations of UCB be protective against the much higher (micromolar) concentrations of reactive oxygen species, especially as $\mathrm{UCB}$ is destroyed in the the process? Several mechanisms may be involved: (1) cyclical regeneration of UCB from biliverdin by the huge excess of cytosolic biliverdin reductase ${ }^{31} ;$ (2) upregulation of haeme oxygenase 1 activity and production of biliverdin (and ultimately UCB) by many stimulii, including oxidative stress ${ }^{27}$; and (3) replenishment of intracellular UCB via uptake after dissociation from the huge reservoir of UCB bound to plasma albumin.

\section{DIFFERENTIAL SUSCEPTIBILITIES TO BILIRUBIN TOXICITY}

Granato and colleagues ${ }^{8}$ reported that rat hepatocytes were unaffected by $B_{f}$ levels approximately three orders of magnitude higher than those that cause cell damage in rodent astrocytes and neurones, ${ }^{3}$ confirming in vivo observations in jaundiced Gunn rats that oxidative phosphorylation and respiration in the liver is unimpaired even in animals with bilirubin encephalopathy and kernicterus. ${ }^{32}$ Apparently, the resistance of the liver to UCB toxicity allows that organ to benefit from the antioxidant properties of the pigment, even at very high UCB levels. In the CNS, regional differences in sensitivity to UCB toxicity are manifested by selective accumulation of UCB only in certain areas of the brain in kernicteric neonates and Gunn rats. ${ }^{5}$ What might account for these differences?

Cells might be protected against accumulation of UCB by transporters that export UCB from the cell back into the blood and by conjugation and/or oxidation of UCB to non-toxic polar derivatives. ${ }^{33}$ When the cellular export and metabolism of UCB are insufficient to keep its total intracellular concentration low, cells might be protected by intracellular proteins that bind $\mathrm{UCB},{ }^{5}$ or by changes in mitochondrial membrane composition. ${ }^{34}$

\section{Transporters}

Both hepatocytes and a variety of CNS cells express two classes of ATP binding cassette $(\mathrm{ABC})$ transporters, multidrug resistance P-glycoproteins (MDR/PGPs) 
and the multidrug resistance associated proteins (MRPs) that can protect cells against accumulation of toxic substances by exporting them back into the plasma. ${ }^{35}$ For unconjugated organic anions, the most important of such exporters are MDRl/mdrl, MRPl/ Mrpl, and MRP3/Mrp3. In the hepatocyte, MDRl is localised apically, and MRPl and 3 basolaterally. ${ }^{35}$ In the cholestatic liver, or after infusion of bile acids, the two Mrps are upregulated to limit accumulation of organic anions into the hepatocyte. ${ }^{36}$ Persistent hyperbilirubinaemia likewise enhances the conjugation and excretion of bilirubin ${ }^{37}$ because binding of UCB to the constitutive androstane receptor upregulates expression of ligandin, UGTIAl, and Mrp2 ${ }^{38}$ It is not known if Mdrla/b or Mrp 1 and 3 are induced by UCB.

$\mathrm{ABC}$ transporters in the capillary endothelium (blood-brain barrier) and/ or the choroid plexus (CP) epithelium (blood-cerebrospinal fluid (CSF) barrier) have been shown to play an important role in limiting the accumulation of organic anions and other small molecules in the CNS and CSF, respectively. ${ }^{33}{ }^{39}$ As yet however there is only weak evidence that UCB is carried by any $\mathrm{ABC}$ transporters. Although some data have suggested that $\mathrm{Mdrla}^{40}$ and $M_{D R} 1^{41}$ limit the accumulation of UCB in CNS cells, these results are inconclusive due to the use of vastly excessive UCB concentrations. ${ }^{9}$ UCB is reportedly a weak substrate for MDRI, ${ }^{42}$ and a role for MRPl in UCB transport has been proposed,,$^{43}{ }^{44}$ but not directly proven. Even if they do transport UCB, the presence of Mrpl and Mdrl in the blood-brain barrier and CP cannot explain the preferential accumulation of UCB in only selected areas of the kernicteric brain. $^{5}$

\section{Conjugation}

Hepatocytes are the major site of bilirubin conjugation, mediated by the specific microsomal UDPGA-bilirubin glucuronosyl transferase, UGTIAl. ${ }^{45}$ In the brain, CP epithelial cells express glucuronosyl transferases for a variety of other organic anions ${ }^{33}$ but UGTIAl has yet to be demonstrated either here or in astrocytes or neurones. Even if present, UGTIAl in the CP could play no role in protecting cultured astrocytes or neurones from UCB toxicity in vitro. In the paper by Granato and colleagues, ${ }^{8}$ bilirubin conjugation by their cultured hepatocytes was extremely low and thus unlikely to account for the resistance of these cells to UCB toxicity.

\section{Oxidation}

In the liver, UCB can be oxidised by the microsomal mixed function monoxygenases, CYPlAl and CYP1A2, but basal expression is low, ${ }^{46}$ although readily inducible by indole-3-carbinol and aryl hydrocarbons. ${ }^{21}{ }^{47}$ In many regions of the brain, low constitutive levels of CYP1A2 are found in neurones and of CYPIAl in the capillary endothelium of the CP and arachnoid..$^{33}$ CYPIAl in liver is transcriptionally induced by elevated UCB levels in jj Gunn rat pups, ${ }^{46}$ mediated by binding of UCB or an oxidation product to the aryl hydrocarbon receptor. ${ }^{48}$ In the latter paper, significant upregulation of CYPIAl occurred after exposure of hepatoma cells to UCB under conditions similar to those used by Granato and colleagues, suggesting that increased oxidation of UCB contributed to the resistance of hepatocytes to UCB toxicity.

\section{Cytosolic binding proteins}

In the hepatocyte, tight binding to two different cytosolic glutathione S-transferases, $\mathrm{Y}$ protein (ligandin) and $\mathrm{Z}$ protein, limits the free cytosolic concentrations of $\mathrm{UCB}^{49}$ and bile salts, ${ }^{505}$ respectively. Ligandin is little expressed in the $\mathrm{CNS}^{52}$ suggesting that the abundance of ligandin in hepatocytes may be a key factor in their greater resistance to UCB toxicity compared with neurones and astrocytes. The reputed antioxidant potency of UCB bound to cytosolic binding proteins ${ }^{23}$ could account for the protective effect of UCB against GCDC mediated apoptosis in hepatocytes, ${ }^{8}$ which is mediated by generation of reactive oxygen species. ${ }^{53}$

Mitochondria from rat livers are themselves more resistant than mitochondria from rat brain to impairment of respiration and oxidative phosphorylation by UCB concentrations in the micromolar range, ${ }^{545}$ even though the net uptake of ${ }^{14} \mathrm{C}$-UCB is similar in both preparations. ${ }^{55}$ These findings suggest that intrinsic changes in the mitochondrial membrane contribute to the greater resistance of hepatocytes to apoptosis caused by hydrophobic bile salts. Additionally, hepatocytes and liver mitochondria from bile duct ligated rats are more resistant to the mitochondrial permeability transition than are the specimens from sham operated controls, and this may be mediated by an increase in mitochondrial cardiolipin content. ${ }^{34}$

\section{CONCLUSIONS}

UCB is a curse at high concentrations, producing apoptosis and cell death, but a boon at more physiological levels, protecting cells against oxidant damage. Differences in organ or cell susceptibility to bilirubin toxicity may be related to differences in expression of $\mathrm{ABC}$ transporters that can export UCB, conjugation and oxidation of the pigment, binding of the pigment to cytosolic binding proteins, and intrinsic differences in the composition of mitochondrial membranes. Conjugation and oxidation convert the pigment to polar non-toxic derivatives which themselves may be antioxidants and/or regulate the metabolism and transport of bilirubin and bile salts. Binding of UCB to ligandin decreases the concentration of the toxic unbound fraction and may enhance the antioxidant potency of the bound fraction. Much additional work, preferably performed with UCB at relevant concentrations, is needed to verify or disprove these possibilities.

\section{ACKNOWLEDGEMENTS}

CT was supported in part by grants from the Italian Ministry for Scientific Research, Fondo Studi Fegato-ONLUS (FCRT 00/01), the Italian Ministry of Health (ICS060.1/ RF98.67), and the University of Trieste.

Gut 2003:52:1668-1670

a...........................

Authors' affiliations

J D Ostrow, Research Service, VA Puget Sound Health Care System-Seattle Division, and $\mathrm{Gl} /$ Hepatology Division, Department of Medicine, University of Washington School of Medicine, Seattle, WA, USA

C Tiribelli, Liver Research Center, AREA Science Park and Department of BBCM, University of Trieste, 34012 Trieste, Italy

Correspondence to: Dr J D Ostrow, Research Service (151L), VA Puget Sound Health Care System-Seattle Division, 1660 South Columbian Way, Seattle, WA 98108-1597, USA; jdostrow@medicine.washington.edu

\section{REFERENCES}

1 Botla R, Spivey JR, Aguilar H, et al. Ursodeoxycholate (UDCA) inhibits the mitochondrial membrane permeability transition induced by glycochenodeoxycholate: a mechanism of UDCA cytoprotection. J Pharmacol Exp Ther 1995;272:930-8.

2 Rodrigues CM, Fan G, Wong PY, et al. Ursodeoxycholic acid may inhibit deoxycholic acid-induced apoptosis by modulating mitochondrial transmembrane potential and reactive oxygen species production. Mol Med 1998:4:165-78.

3 Ostrow JD, Pascolo L, Tiribelli C. Reassessment of the unbound concentrations of unconjugated bilirubin in relation to neurotoxicity in vitro. Pediatr Res 2003:54:98-104.

4 Beuers U, Boyer JL, Paumgartner G Ursodeoxycholic acid in cholestasis: potentia mechanism for action and therapeutic applications. Hepatology 1998;28: 1449-53.

5 Gourley GR. Bilirubin metabolism and kernicterus. Adv Pediatr 1997;44:173-229.

6 Rodrigues CMP, Sola S, Brites D. Bilirubin induces apoptosis via the mitochondrial pathway in developing rat brain neurons. Hepatology 2002:35: 1186-95.

7 Silva RFM, Rodrigues CMP, Brites D. Bilirubininduced apoptosis in cultured rat neural cells is aggravated by chenodeoxycholic acid but prevented by ursodeoxycholic acid. J Hepatol 2001;34:402-8.

8 Granato A, Gores G, Vilei MT, et al. Bilirubin inhibits bile acid induced apoptosis in rat hepatocytes. Gut 2003:52:1774-8.

9 Ostrow JD, Tiribelli C. Editorial: New concepts in bilirubin neurotoxicity and the need for studies at 
clinically relevant bilirubin concentrations. J Hepatol 2001;34:467-70.

10 Ostrow JD. In vitro studies of bile salt toxicity. Hepatology 1994;19:1538-9.

11 Hahm JS, Ostrow JD, Mukerjee P, et al. Ionization and self-association of unconjugated bilirubin, determined by rapid solvent partition from chloroform, with further studies of bilirubin solubility. J Lipid Res 1992;33:1 123-37.

12 Brodersen R, Stern L. Aggregation of bilirubin in injectates and incubation media: its significance in experimental studies of CNS toxicity. Neuropediatrics 1987;18:34-6.

13 Mukerjee P, Ostrow JD, Tiribelli C. Low solubility of unconjugated bilirubin in dimethylsulfoxidewater systems: implications for $\mathrm{pK}_{0}$ determinations. BMC Biochemistry 2002;3:17.

14 McDonagh AF, Assisi F. The ready isomerization of bilirubin-IX $\alpha$ in aqueous solution. Biochem $J$ 1972; 129:797-800.

15 Del Vecchio S, Ostrow JD, Mukerjee P, et al. Method for removal of surface-active impurities and calcium from conjugated bile salt preparations: comparison with silicic acid chromatography. J Lipid Res 1995;36:2639-50.

16 Hirsch JI, Ahrens EH Jr. The separation of complex lipid mixtures by the use of silicic acid chromatography. J Biol Chem 1958;233:311-20

17 Stocker R, Yamamoto Y, McDonagh AF, et al. Bilirubin is an antioxidant of possible physiological importance. Science 1987;235: 1043-6.

18 Stocker R, Peterhans E. Synergistic interaction between vitamin $E$ and the bile pigments bilirubin and biliverdin. Biochim Biophys Acta 1989; 1002:238-44

19 Tomaro ML, Batlle AMD. Bilirubin: its role in cytoprotection against oxidative stress. Int J Biochem Cell Biol 2002;34:216-20.

20 Dore S, Snyder SH. Neuroprotective action of bilirubin against oxidative stress in primary hippocampal cultures. Ann NY Acad Sci 1999;890: 167-72.

21 Ostrow JD Kapitulnik J. Alternate pathways of heme and bilirubin metabolism. In: Ostrow JD, ed. Bile pigments and jaundice: molecular metabolic and medical aspects. New York: Marcel Dekker, 1986:421-38.

22 Stocker R, Glazer AN, Ames BN. Antioxidant activity of albumin-bound bilirubin. Proc Natl Acad Sci U S A 1987;84:5918-22.

23 Brass CA, Wrchota EM, Gollan JL. Bilirubin is a potent inhibitor of lipid peroxidation in hepatic microsomal membranes. Hepatology 1989; 10:609.

24 Ahlfors CE. Bilirubin-albumin binding and free bilirubin. J Perinatol 2001;21(suppl 1):S40-2.

25 Weisiger RA, Ostrow JD, Koehler RK, et al. Affinity of human serum albumin for bilirubin varies with albumin concentration and buffer composition: results of a novel ultrafiltration method. J Biol Chem 2001;276:29953-60.

26 Zucker SD, Goessling W, Hoppin AG. Unconjugated bilirubin exhibits spontaneous diffusion through model lipid bilayers and native hepatocyte membranes. J Biol Chem 1999;274: 10852-62.

27 Ryter SW, Tyrrell RM. The heme synthesis and degradation pathways: role in oxidant sensitivity. Heme oxygenase has both pro- and antioxidant properties. Free Radic Biol Med 2000;28:289-309.

28 Dore S, Takahashi M, Ferris CD, et al. Bilirubin formed by activation of heme oxygenase- 2 , protects neurons against oxidative stress injury. Proc Natl Acad Sci U S A 1999;96:2445-50.

29 Clark JE, Foresti R, Green CJ, et al. Dynamics of haem oxygenase-1 expression and bilirubin production in cellular protection against oxidative stress. Biochem J 2000;348:615-19.

30 Zuckerbraun BS, Billiar TR. Heme oxygenase-1: a cellular Hercules. Hepatology 2003;37:742-4.

31 Barañano $D E$, Rao $M$, Ferris $C D$, et al. From the Cover: Biliverdin reductase: A major physiologic cytoprotectant. Proc Natl Acad Sci U S A 2002;99:16093-8.

32 Schenker S, McCandless DW, Wittgenstein E. Studies in vivo of the effect of unconjugated bilirubin on hepatic phosphorylation and respiration. Gut 1966;7:409-14.

33 Ghersi-Egea JF, Strazielle N. Brain drug delivery, drug metabolism, and multidrug resistance at the choroid plexus. Microsc Res Tech 2001;52:83-8.

34 Lieser MJ, Park J, Natori S, et al. Cholestasis confers resistance to the rat liver mitochondrial permeability transition. Gastroenterology 1998; 115:693-701.

35 Borst P, Oude Elferink R. Mammalian abc transporters in health and disease. Annu Rev Biochem 2002:71:537-92.

36 Kullak-Ublick GA, Beuers U, Paumgartner G. Hepatobiliary transport. J Hepatol 2000;32(suppl 1):3-18.

37 Munoz ME, Esteller A, Gonzalez J. Substrate induction of bilirubin conjugation and biliary excretion in the rat. Clin Sci 1987:73:371-5.

38 Huang W, Zhang J, Chua SS, et al. Induction of bilirubin clearance by the constitutive androstane receptor (CAR). Proc Natl Acad Sci U S A 2003.100:4156-61.

39 Wiinholds J, de Lange EC, Scheffer GL, et al. Multidrug resistance protein 1 protects the choroid plexus epithelium and contributes to the blood-cerebrospinal fluid barrier. J Clin Invest 2000; 105:279-85.

40 Watchko JF, Daood MJ, Hansen TWR. Brain bilirubin content is increased in P-glycoproteindeficient transgenic null mutant mice. Pediatr Res 1998:44:763-6

41 Watchko JF, Ziegler J, Daood MJ, et al. Inhibition of human MDR1 P-glycoprotein increases bilirubin-induced cell apoptosis in vitro. Pediatr Res 2003;53:400A

42 Gosland MP, Brophy NA, Duran GE, et al. Bilirubin: a physiological substrate for the multidrug transporter. Proc Am Assn Cancer Res $1991 ; 32: 426$.

43 Petrovic S, Pascolo L, Gallo R, et al. The products of YCF1 and YLLO15w (BPT1) cooperate for the ATP-dependent vacuolar transport of unconjugated bilirubin in Saccharomyces cerevisiae. Yeast 2000;16:561-71.

44 Pascolo L, Fernetti C, Crivellato E, et al. Mechanisms for the transport of unconjugated bilirubin in human trophoblastic BeWo cells. FEBS Lett 2001;495:94-9.

45 Strassburg CP, Manns MP. Jaundice, genes and promoters. J Hepatol 2000;33:476-9.

46 Kapitulnik J, Gonzalez FJ. Marked endogenous activation of the CYP1A1 and CYP1A2 genes in the congenitally jaundiced Gunn rat. Mol Pharmacol 1993;43:722-5.

47 Jorritsma U, Schrader E, Klaunick G, et al Monitoring of cytochrome P-450 1A activity by determination of the urinary pattern of caffeine metabolites in Wistar and hyperbilirubinemic Gunn rats. Toxicology 2000;144:229-36.

48 Phelan D, Winter GM, Rogers WJ, et al. Activation of the Ah receptor signal transduction pathway by bilirubin and biliverdin. Arch Biochem Biophys 1998;357:155-63.

49 Zucker SD, Gössling W, Ransil BJ, et al. Influence of glutathione S-transferase B (ligandin) on the intermembrane transfer of bilirubin-implications for the intracellular transport of nonsubstrate ligands in hepatocytes. $J$ Clin Invest 1995:98:1927-35.

50 Takikawa H, Sugiyama Y, Kaplowitz N. Binding of bile acids by glutathione S-transferases from rat liver. J Lipid Res 1986;27:955-66.

51 Stolz A Sugiyama Y, Kuhlenkamp J, et al. Cytosolic bile acid binding protein in rat liver: radioimmunoassay, molecular forms, developmental characteristics and organ distribution. Hepatology 1986:6:433-9.

52 Abramovitz $\mathrm{M}$, Homma $\mathrm{H}$, Ishigaki S, et al. Characterization and localization of glutathione$\mathrm{S}$-transferases in rat brain and binding of hormones, neurotransmitters, and drugs. J Neurochem 1988:50:50-7.

53 Sokol RJ, Devereaux M, Khandwala R, et al Evidence for involvement of oxygen free radicals in bile acid toxicity to isolated rat hepatocytes. Hepatology 1993; 17:869-81.

54 Mustafa MG, Cowger ML, King TE. Effects of bilirubin on mitochondrial reactions. J Biol Chem 1969;244:6403-14.

55 Menken M, Waggoner JG, Berlin NI. The influence of bilirubin on oxidative phosphorylation and related reactions in brain and liver mitochondria: effects of protein-binding. J Neurochem 1966;13:1241-8. 